\title{
A Survey of the Status of Awareness of Lymphedema in Breast Cancer Patients in Busan-Gyeongnam, Korea
}

\author{
Jong Kyoung Choi, $\mathrm{MD}^{1}$, Hui Dong Kim, $\mathrm{MD}^{1}$, Young Joo Sim, MD, $\mathrm{PhD}^{1}$, Ghi Chan Kim, MD, PhD ${ }^{1}$, \\ Dong Kyu Kim, $\mathrm{MD}^{1}$, Byeng Chul Yu, MD, $\mathrm{PhD}^{2}$, Si-Sung Park, MD, PhD ${ }^{3}$, Ho Joong Jeong, MD, $\mathrm{PhD}^{1}$
}

Departments of ${ }^{1}$ Physical Medicine \& Rehabilitation, ${ }^{2}$ Preventive Medicine, and ${ }^{3}$ Psychiatry, Kosin University College of Medicine, Busan, Korea

Objective To support the establishment of lymphedema education plans and the actual practice of education by investigating the current lymphedema awareness status of Korean breast cancer patients.

Methods A cross-sectional population survey was conducted in 116 breast cancer patients in the Busan-Gyeongnam area. The survey included questions regarding demographic characteristics, breast cancer-related lymphedema (BCRL) risk factors, and characteristics and treatments of the disease. Some of the items were scored to determine the level of awareness. The items that affect the awareness of lymphedema were investigated by statistical analysis. Results Eighty-one of the 116 patients answered that they had heard of lymphedema, and 30 of them (25.86\%) had received explanations about the possibility of lymphedema before surgery. Only 20 patients (17.25\%) knew that lymphedema is not a completely curable disease, 24 patients $(20.68 \%)$ thought that lymphedema does not require any treatment, and only 56 patients (48.27\%) knew that lymphedema is treated in the Department of Rehabilitation Medicine. The main factors that affected patients' awareness of lymphedema were their age, chemotherapy, duration of breast cancer, and lymphedema treatment history.

Conclusion The majority of survey participants who were breast cancer patients either lacked awareness of BCRL or had false ideas about it, indicating the inadequate level of education provided for lymphedema. In the case of breast cancer diagnosis, early and continuous education for future management is essential, and the framework for the provision of education including education protocols related to age, disease duration, and lymphedema treatment is needed.

Keywords Lymphedema, Breast neoplasms, Education

() This is an open-access article distributed under the terms of the Creative Commons Attribution Non-Commercial License (http://creativecommons.org/ licenses/by-nc/4.0) which permits unrestricted noncommercial use, distribution, and reproduction in any medium, provided the original work is properly cited. Copyright $\odot 2015$ by Korean Academy of Rehabilitation Medicine 


\section{INTRODUCTION}

Lymphedema is a chronic disease involving the accumulation of protein-rich fluid in the interstitial space due to abnormal lymphatic circulation [1]. Lymphedema can be separated into primary and secondary types, and although it is induced by a variety of causes, it is most commonly caused by breast cancer itself or as a complication of breast cancer treatment [2]. The lymphedema that is caused by breast cancer itself or due to breast cancer treatments is called breast cancer-related lymphedema (BCRL), and its incidence is reported to be $6 \%-83 \%$, indicating that different studies have found vastly different results [3]. BCRL is a serious complication that impairs the upper extremity functions and quality of life of breast cancer patients. The progression of BCRL is related to various risk factors including surgery, radiation therapy, chemotherapy, diagnostic time, pathological lymph node condition, infection, and weight gain [4-6]. Prevention by risk factor management is an important part of lymphedema treatment [7], and several studies have reported that the treatment effects, prognosis of BCRL, and the quality of life of breast cancer patients are greatly affected by the patients' awareness of BCRL and its risk factors [810]. Although there have been studies on BCRL-related awareness, risk factors, and education in other countries, no evaluation of the BCRL-related awareness level in Korean breast cancer patients has been conducted. Thus, the appropriateness of current BCRL-related education or of early detection of lymphedema cannot be determined. Therefore, this study was conducted in order to investigate the level of awareness of BCRL in breast cancer patients, and to provide the basic data needed to establish a proper education time and method.

\section{MATERIALS AND METHODS}

\section{Subjects}

This study conducted a cross-sectional population survey from September 2012 to August 2013 on 125 breast cancer patients to investigate their level of awareness of BCRL. The recruited subjects included 70 patients from the Breast Cancer Patient United known as Pink Ribbon, and 55 outpatients and inpatients of the breast clinic that were not members of Pink Ribbon. The authors determined the number of samples for this study by referring to the sample size of previous studies [10-14] conducted in other countries without calculation. Among the recruited subjects, 4 refused to participate in the survey and 5 did not completely answer the questionnaire, thus they were excluded from the study; a total of 116 subjects' answers were analyzed.

\section{Questionnaire and methods}

There were 24 questions in the survey including demographic characteristics such as body weight, height, age, dominant hand, basic medical history information, such as breast cancer diagnosis and surgery dates, site, method, whether the patient received radiation therapy or chemotherapy before and after surgery, whether lymphedema was diagnosed and treated, whether the patient had heard about lymphedema, whether the patient had received an explanation from medical staff on the possibility of lymphedema before and after breast cancer surgery, awareness of generally known risk factors of lymphedema (upper limb hygiene, mild trauma, tight clothing or wearing bracelets, excessive use of upper extremities, weight gain) $[2,3,6]$, and awareness of lymphedema-related treatment (whether lymphedema needs to be medically treated, if it is curable, which medical department treats it, what exercise is helpful). All of the contents and survey questions in the present study were investigated to meet with the patient directly without checking the patients' medical records (Table 1).

Each answer to a question was analyzed in total ratio, and among 24 questions in the survey, 3 questions asked whether they had received any general information on lymphedema from medical staff, 9 questions were on the risk factors and treatment of lymphedema. A total of 12 questions measured the awareness of lymphedema. Each question was scored. For the comparison of each question item, a Student t-test was used, the awareness level was compared and analyzed, and multiple regression analysis was performed, including age and disease duration, to analyze the influence of the factors that affect the awareness level.

\section{RESULTS}

\section{General characteristics of subjects}

The average age of the 116 subjects in the present study was $51.96 \pm 7.63$ (range, 31-67 years). The average body 
Table 1. Status of education \& awareness of the risk factors and management of BCRL in breast cancer patients

No $(\%)$

\section{Status of education about BCRL}

Do you know about lymphedema?

Yes

No

Did you receive an explanation about the possibility of lymphedema by a medical team member before surgery?

Yes

No

Did you receive an explanation about the possibility of lymphedema by medical a team member after surgery?

Yes

No

Level of awareness of risk factors and management of BCRL

Is it possible that poor hygiene of an upper limb can increase the risk of lymphedema occurrence?

Yes

No

Is it possible that trauma of an upper limb can increase the risk of lymphedema occurrence?

Yes

No

Is it possible that constriction of an upper limb by a bracelet or tight shirt can increase the risk of lymphedema occurrence?

Yes

No

Is it possible that overuse of an upper limb can increase the risk of lymphedema occurrence?

Yes

No

Is it possible that weight gain can increase the risk of lymphedema occurrence? Yes

No

Is lymphedema a disease that should be treated?

Yes

No

Is lymphedema a disease that cannot be completely cured?

Completely curable

Not completely curable

Which activity is helpful to reduce the risk of lymphedema occurrence?

Golf

Tennis (using affected upper limb)

$2(1.72)$

Swimming

$50(43.10)$

Ping-pong (using affected upper limb)

$3(2.59)$

Hard weight training

$27(23.27)$

No idea

Which department can manage lymphedema?

General surgery

Family medicine

Physical medicine and rehabilitation

Orthopedic surgery

No idea

BCRL, breast cancer-related lymphedema. 
mass index was $36.90 \pm 5.58 \mathrm{~kg} / \mathrm{m}^{2}$. Among the subjects, 106 were right-handed, 10 were left-handed, 116 had had a mastectomy, and among them, 47 had had a left mastectomy, 63 had had a right mastectomy, and 6 had had a bilateral mastectomy. Eighty-three subjects (69.75\%) had had armpit lymph node dissection. Seventy-five patients (64.65\%) underwent chemotherapy, and 70 patients (60.34\%) underwent radiation therapy. Among the participants, 30 (25.86\%) were diagnosed with BCRL, and 26 of these $(22.41 \%)$ received treatment for BCRL.

\section{Awareness of the risk factors of BCRL}

Among the breast cancer patients, 65 (56.03\%) and 84 (72.41\%) answered that poor upper limb hygiene and mild trauma were risk factors of lymphedema, respectively. In addition, $83(71.55 \%)$ answered that tight shirts or wearing bracelets are risk factors, $79(68.10 \%)$ answered that an excessive use of the affected upper limb can cause lymphedema, and 64 (55.17\%) answered that weight gain can cause lymphedema. Regarding the question asking the participants to choose the proper exercise for breast cancer patients, 66 (56.9\%) chose 'strenuous exercise' or 'I'm not sure.' Overall, when answering the questions on risk factors, the lymphedema-diagnosed group showed a greater awareness of the risk factors of lymphedema compared with the patient group that had never been diagnosed with lymphedema. However, with respect to the question asking the participants to choose the proper exercise that is helpful for lymphedema patients, the lymphedema-diagnosed group chose 'strenuous exercise' more often than the group that had never been diagnosed with lymphedema.

\section{Awareness of the treatment of BCRL}

For the question regarding the level of awareness about the treatment for lymphedema, 24 breast cancer patients $(20.68 \%)$ were found to have no idea that lymphedema is a disease that needs to be medically treated, and 96 (82.75\%) answered that lymphedema is curable. Among the patients who were diagnosed with lymphedema, $72 \%$ thought that it is curable (Table 2). In addition, 29 (25\%) answered 'I'm not sure.' for the question regarding which medical department treats lymphedema, and 31 (26.73\%) chose orthopedic surgery or general surgery, not rehabilitation medicine. This shows that more than half of the patients did not know that lymphedema is treated in the department of rehabilitation medicine.

\section{Influence of each factor on awareness}

The results of the univariate analysis of each factor that affected awareness showed that the patients from the Pink Ribbon association, the patients who had had lymph node dissection, chemotherapy or radiation therapy, and a previous lymphedema diagnosis and treatment, all had a significantly higher awareness of BCRL (Table 3).

In addition, multivariate analysis including the factors used for the above univariate analysis, in addition to the patients' age and duration of illness (time since breast cancer diagnosis), showed that the younger patients, who had had chemotherapy and treatment for lymphedema and a long duration of illness showed a significantly increased awareness of BCRL. Among these, the strongest influences on the awareness of lymphedema were found to be ranked in the following order: lymphedema treatment received at a hospital, chemotherapy, duration of illness, and age (Table 2).

Table 2. Association of lymphedema awareness in breast cancer patients with variables by multiple regression analysis

\begin{tabular}{lrrrrc}
\hline \multicolumn{1}{c}{ Variable } & B & SD & $\boldsymbol{\beta}$ & \multicolumn{1}{c}{ t } & p-value \\
\hline Age & -0.055 & 0.027 & -0.163 & -2.023 & 0.046 \\
Breast cancer association patients (Pink Ribbon) & 0.315 & 0.466 & 0.060 & 0.677 & 0.500 \\
Lymph node dissection surgery & 0.171 & 0.489 & 0.030 & 0.350 & 0.727 \\
Chemotherapy & 1.890 & 0.791 & 0.354 & 2.391 & 0.019 \\
Radiotherapy & 0.326 & 0.757 & 0.063 & 0.431 & 0.667 \\
Lymphedema diagnosis & -1.114 & 1.118 & -0.191 & -0.997 & 0.321 \\
Lymphedema treatment & 2.687 & 1.187 & 0.439 & 2.263 & 0.026 \\
Disease duration & 0.300 & 0.124 & 0.220 & 2.417 & 0.017 \\
\hline
\end{tabular}

$\mathrm{R}^{2}=0.399$, adjusted $\mathrm{R}^{2}=0.354$. 
Table 3. Comparison of each question item

\begin{tabular}{llcc}
\hline & & $\begin{array}{c}\text { Awareness of lymphedema } \\
\text { (total score, 12 points) }\end{array}$ & p-value $^{\text {a) }}$ \\
\hline Chemotherapy & Yes (75) & $7.56 \pm 2.05$ & 0.000 \\
Radiotherapy & No (41) & $5.00 \pm 2.58$ & 0.000 \\
\hline Lymphedema diagnosis & Yes (70) & $7.48 \pm 2.09$ & 0.000 \\
\hline Members of the breast cancer association (Pink Ribbon) & No (46) & $5.39 \pm 2.70$ & 0.005 \\
& Yes (30) & $8.10 \pm 2.47$ & 0.026 \\
\hline Lymph node dissection & No (86) & $6.15 \pm 2.41$ & $7.18 \pm 2.40$ \\
\hline
\end{tabular}

${ }^{a)}$ Student t-test.

\section{DISCUSSION}

Lymphedema following breast cancer surgery can cause a number of serious clinical or psychological problems. According to Tobin et al. [15], 46\% of 50 lymphedema patients have a functional disability. Lymphedema is one of the most serious complications that can result from breast cancer treatment, which can clinically induce a limited range of motion and pain in the affected upper limb, weakness or tightness, and can cause secondary psychological problems such as anxiety, depression, sexual dysfunction, social avoidance, and aggravation of existing psychological illnesses [8,15-18]. There is currently no medical cure for lymphedema; therefore, it is important to prevent lymphedema by educating patients on the avoidance of risk factors and how to observe their own arms for early diagnosis and treatment $[7,10,19]$. Woods [8] conducted a survey of 40 breast cancer patients investigated with semi-structured interviews, Psychosocial Adjustment to Illness Scale, and limb volume measurement, and reported that increasing awareness has the benefit of maximum improvement and long-term control for patients with lymphedema. In addition, Sherman and Koelmeyer [11,12], reported that information delivery on lymphedema in a brochure format or via clinic staff plays an important role in minimizing the risk of lymphedema. Moreover, patients' higher awareness of lymphedema helps more effective early diagnosis and treatment of lymphedema. Fu et al. [13] also reported that education on lymphedema strengthens patients' self-management. Medical institutions in Korea are educating patients to be more aware of lymphedema, yet there have been no prior studies conducted on the patients' level of awareness or knowledge on the risk factors and treatments of lymphedema in Korea. Previous studies [10,11-14] conducted in other countries have the disadvantages of a small sample number of samples or survey over the phone. The present study increased the number of samples compared with the number of patients, and met with patients in person to survey them, in order to help to plan and conduct education on lymphedema for breast cancer patients in Korea.

Among the recruited breast cancer patients in the present study, barely more than half of the patients were aware of lymphedema as a disease, and only 30 patients (25.9\%) received explanations about the possibility of lymphedema from the medical staff before surgery. The patients who were diagnosed with lymphedema showed a higher awareness regarding all of the items on the questionnaire. This means that prevention and management of lymphedema needs be carried out in the early stages and not conducted after exposure to various risk factors or after the diagnosis of lymphedema.

In addition, $20.69 \%$ of the patients who participated in the survey answered that lymphedema is a disease that does not require medical treatment, $82.75 \%$ answered 
that lymphedema is a curable disease, and notably, 21 out of the 30 patients (70\%) who were diagnosed with lymphedema also answered that lymphedema is curable. Regarding the question asking whether they knew which medical department treats lymphedema, $51.73 \%$ of the patients either chose departments other than rehabilitation medicine or answered 'I'm not sure.'; only 19 out of the 30 patients who were diagnosed with lymphedema (63\%) answered 'Rehabilitation Medicine'. According to the results above, the patients seemed not to have the proper information even after the diagnosis of lymphedema. In addition, for the questions regarding the risk factors of lymphedema, such as weight gain, hygiene, or aggravated lymphedema, patients showed a relatively lower level of awareness (55.6\%) compared with that of the other items ( $70 \%$ on average) on the questionnaire.

Multiple regression analysis results show that the factors that affect this awareness are chemotherapy, lymphedema treatment, duration of illness, and age. In the case of those who underwent chemotherapy and lymphedema treatment with a long duration of illness, the patients may have had greater exposure to other patients or medical staff, and this may have had a positive effect on their awareness of lymphedema. In addition, considering that younger patients also exhibited a higher awareness, cognitive decline in the aging population may have affected the level of awareness of lymphedema. The above results suggest that the quality and timing of the current education on lymphedema are problematic.

Therefore, for proper management of lymphedema, education protocols considering age, duration of illness, and whether the patient has had chemotherapy need to be developed. In addition, although it did not significantly affect the level of awareness of lymphedema, the patients that were members of a breast cancer patient association and those who were diagnosed with lymphedema showed a difference in awareness level. More active education for breast cancer patients to approach their disease is necessary. In addition, continuous exposure to information on lymphedema can help to improve the patients' awareness level and approach towards lymphedema, even for those who have already been educated.

The limitations of the present study were the small number of subjects, and the fact that the difference in the level of awareness was not investigated before and after education on lymphedema, therefore the direct associa- tion between education and awareness level could not be proven. Moreover, the degree that education affects awareness could not be examined due to different learning abilities and educational backgrounds of the patients. In addition, the patients that were not diagnosed with lymphedema were not examined for the presence of lymphedema, and the investigation of the difference in the level of awareness of lymphedema and access to the diagnosis and treatment for lymphedema was not accurately conducted. Future studies need to recruit a greater number of subjects and work towards understanding the relationship between education and changes in the awareness level of patients.

\section{CONFLICT OF INTEREST}

No potential conflict of interest relevant to this article was reported.

\section{REFERENCES}

1. Jeong HJ, Eom MS, Choi SB, Kim DS, Kang KM. Risk factors for developing upper limb lymphedema following breast cancer surgery. J Korean Acad Rehabil Med 2008;32:95-9.

2. Jeong HJ, Kim GC, Roh KH, Shin DH, Lee CH. The risk factors of lymphedema after breast cancer surgery. J Korean Acad Rehabil Med 2002;26:475-9.

3. Clark B, Sitzia J, Harlow W. Incidence and risk of arm oedema following treatment for breast cancer: a three-year follow-up study. QJM 2005;98:343-8.

4. McLaughlin SA, Bagaria S, Gibson T, Arnold M, Diehl $\mathrm{N}$, Crook J, et al. Trends in risk reduction practices for the prevention of lymphedema in the first 12 months after breast cancer surgery. J Am Coll Surg 2013; 216:380-9.

5. Park JE, Jang HJ, Seo KS. Quality of life, upper extremity function and the effect of lymphedema treatment in breast cancer related lymphedema patients. Ann Rehabil Med 2012;36:240-7.

6. Kocak Z, Overgaard J. Risk factors of arm lymphedema in breast cancer patients. Acta Oncol 2000;39:389-92.

7. Soran A, D'Angelo G, Begovic M, Ardic F, Harlak A, Samuel Wieand $\mathrm{H}$, et al. Breast cancer-related lymphedema: what are the significant predictors and how they affect the severity of lymphedema? Breast J 2006; 
12:536-43.

8. Woods M. Patients' perceptions of breast-cancer-related lymphoedema. Eur J Cancer Care (Engl) 1993;2: 125-8.

9. Morgan PA, Franks PJ, Moffatt CJ. Health-related quality of life with lymphoedema: a review of the literature. Int Wound J 2005;2:47-62.

10. Bosompra K, Ashikaga T, O’Brien PJ, Nelson L, Skelly J, Beatty DJ. Knowledge about preventing and managing lymphedema: a survey of recently diagnosed and treated breast cancer patients. Patient Educ Couns 2002;47:155-63.

11. Sherman KA, Koelmeyer L. Psychosocial predictors of adherence to lymphedema risk minimization guidelines among women with breast cancer. Psychooncology 2013;22:1120-6.

12. Sherman KA, Koelmeyer L. The role of information sources and objective risk status on lymphedema riskminimization behaviors in women recently diagnosed with breast cancer. Oncol Nurs Forum 2011;38:E2736.

13. Fu MR, Chen CM, Haber J, Guth AA, Axelrod D. The effect of providing information about lymphedema on the cognitive and symptom outcomes of breast cancer survivors. Ann Surg Oncol 2010;17:1847-53.
14. Kwan ML, Shen L, Munneke JR, Tam EK, Partee PN, Andre M, et al. Patient awareness and knowledge of breast cancer-related lymphedema in a large, integrated health care delivery system. Breast Cancer Res Treat 2012;135:591-602.

15. Tobin MB, Lacey HJ, Meyer L, Mortimer PS. The psychological morbidity of breast cancer-related arm swelling: psychological morbidity of lymphoedema. Cancer 1993;72:3248-52.

16. Maunsell E, Brisson J, Deschenes L. Arm problems and psychological distress after surgery for breast cancer. Can J Surg 1993;36:315-20.

17. Sneeuw KC, Aaronson NK, Yarnold JR, Broderick M, Regan J, Ross G, et al. Cosmetic and functional outcomes of breast conserving treatment for early stage breast cancer. 2 . Relationship with psychosocial functioning. Radiother Oncol 1992;25:160-6.

18. Passik S, Newman M, Brennan M, Holland J. Psychiatric consultation for women undergoing rehabilitation for upper-extremity lymphedema following breast cancer treatment. J Pain Symptom Manag 1993;8: 22633.

19. Morrell RM, Halyard MY, Schild SE, Ali MS, Gunderson LL, Pockaj BA. Breast cancer-related lymphedema. Mayo Clin Proc 2005;80:1480-4. 